\title{
Ginkgolide J protects human synovial cells SW982 via suppression of p38-dependent production of pro-inflammatory mediators
}

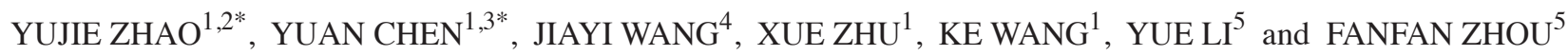 \\ ${ }^{1}$ NHC Key Laboratory of Nuclear Medicine, Jiangsu Key Laboratory of Molecular Nuclear Medicine, \\ Jiangsu Institute of Nuclear Medicine, Wuxi, Jiangsu 214063; ${ }^{2}$ The First Clinical School, Wenzhou Medical University, \\ Wenzhou, Zhejiang 325035; ${ }^{3}$ Department of Radiopharmaceuticals, School of Pharmacy, Nanjing Medical University, \\ Nanjing, Jiangsu 211166; ${ }^{4}$ Department of Rheumatology, Shanghai East Hospital, Tongji University School of Medicine, \\ Shanghai 200120, P.R. China; ${ }^{5}$ Faculty of Pharmacy, The University of Sydney, Sydney, NSW 2006, Australia
}

Received August 13, 2020; Accepted February 8, 2021

DOI: $10.3892 / \mathrm{mmr} .2021 .12194$

\begin{abstract}
Fibroblast-like synoviocytes (FLS) in the synovial lining play a key role in the pathological process of rheumatoid arthritis (RA), which produce pro-inflammatory mediators to perpetuate inflammation and proteases to contribute to cartilage destruction. Ginkgolide $\mathrm{J}(\mathrm{GJ})$ is a subclass of ginkgolides (GGs) that exhibits anti-inflammatory activity. In the present study, the protective effect of GJ on lipopolysaccharide (LPS)-treated human synovial cells SW982 and its related mechanisms were investigated using various methods, including ELISA, Griess assay, western blotting, immunofluorescence analysis and p38 kinase activity assay. The results revealed that GJ pretreatment significantly attenuated LPS-induced excess production of pro-inflammatory mediators in SW982 cells via suppression of tumor necrosis factor- $\alpha$ /interleukin (IL)- $1 \beta / \mathrm{IL}-18 / \mathrm{NF}-\kappa \mathrm{B} / \mathrm{NLR}$ family pyrin domain containing 3 , prostaglandin E2/cyclooxygenase-2 and inducible nitric oxide synthase/nitric oxide signaling. Mechanistic studies revealed that $\mathrm{p} 38$ activation contributed to the LPS-induced inflammatory response, and GJ pretreatment dose-dependently attenuated p38 activation, indicating that the suppressive effect of GJ was achieved by targeting p38
\end{abstract}

Correspondence to: Dr Jiayi Wang, Department of Rheumatology, Shanghai East Hospital, Tongji University School of Medicine, 150 Ji Mo Road, Shanghai 200120, P.R. China

E-mail: doctorwjy@outlook.com

Professor Xue Zhu, NHC Key Laboratory of Nuclear Medicine, Jiangsu Key Laboratory of Molecular Nuclear Medicine, Jiangsu Institute of Nuclear Medicine, 20 Qianrong Road, Wuxi, Jiangsu 214063, P.R. China

E-mail: zhuxue@jsinm.org

${ }^{*}$ Contributed equally

Key words: rheumatoid arthritis, fibroblast-like synoviocytes, Ginkgolide J, p38 signaling signaling. These findings may contribute to the prevention and treatment of RA.

\section{Introduction}

Chronic joint inflammation is the hallmark of rheumatoid arthritis (RA), the most common type of autoimmune disease $(1,2)$. Although the origin of the disease remains unclear, the immune system is known to affect the lining of joints and cause a painful swelling that may eventually result in bone erosion and joint deformity (3). The synovial lining of diarthrodial joints is the site of the initial inflammatory process, and it contains abundant cytokines and chemokines produced by several immune cell types (4). Fibroblast-like synoviocytes (FLS) are of mesenchymal origin in the synovial lining, and RA FLS are important mediators of joint destruction that have the ability to invade adjacent collagenous structures and secrete factors that promote inflammation, neovascularization, and cartilage degradation (5). Thus, strategies to control the inflammatory effect of FLS may contribute to the prevention and treatment of RA.

Ginkgo biloba extract (GBE), one of the most common industrialized herbal medicines, has been clinically used in the treatment of cardiovascular, cerebrovascular, and neurological disorders due to its anti-inflammatory, antioxidant and anti-apoptotic activities (6-8). The major identified active ingredients of GBE include ginkgo flavonol glycosides (GFGs) and ginkgolides (GGs) (9). GGs consist of ginkgolide A (GA), ginkgolide B (GB), ginkgolide C (GC), ginkgolide J (GJ), and ginkgolide $\mathrm{K}(\mathrm{GK})$ (10). GA has been revealed to suppress the expression of pro-inflammatory mediators [cyclooxygenase-2 (COX-2) and nitric oxide (NO)] and pro-inflammatory cytokines [tumor necrosis factor (TNF)- $\alpha$, interleukin (IL)- 6 and IL-1 $\beta$ ] in LPS-treated mouse and human macrophages (11). Other CGs have exhibited anti-platelet-activating, anti-apoptotic, anti-oxidative, neurotrophic and neuroimmunomodulatory effects by inhibition of the mitogen-activated protein kinase (MAPK) and nuclear factor $-\kappa B(N F-\kappa B)$ signaling pathways (12). Among these CGs, GB has the most obvious pharmacological properties, while little is known about GJ $(13,14)$. Vitolo et al $(15)$ reported that GJ is capable of 
inhibiting the cell death of rodent hippocampal neurons caused by $A \beta(1-42)$. However, the pharmacological effect of GJ on RA has yet to be investigated. Bacterial LPS is capable of eliciting a strong immune response and frequently used to induce symptoms of RA (16). In the present study, the protective effect of GJ against inflammation induced by LPS in human synovial cells SW982 as well as the underlying mechanisms were investigated.

\section{Materials and methods}

Reagents. GJ was provided from Wanbangde Pharmaceutical Group Co., Ltd. LPS, DMSO and DAPI were purchased from Sigma-Aldrich (Merck KGaA). Antibodies were purchased from Santa Cruz Biotechnology, Inc., and Abcam. The antibodies used in the present study were as follows: COX-2 (cat. no. ab169782), inducible nitric oxide synthase (iNOS; cat. no. ab178945), NLR family pyrin domain containing 3 (NLRP3; cat. no. ab263899) and caspase-1 (cat. no. ab207802; all 1:1,000; all from Abcam), IL-1 $\beta$ (cat. no. sc-515598), phosphorylated (p)-p38 (cat. no. sc-166182), p38 (cat. no. sc-7972) and GAPDH (cat. no. sc-365062; all 1:1,000; all from Santa Cruz Biotechnology, Inc.). Other reagents were purchased from Beyotime Institute of Biotechnology and Sangon Biotech Co., Ltd.

Cell culture and treatment. Human synovial cells SW982 were purchased from American Type Culture collection and maintained in DMEM with 10\% fetal bovine serum (FBS) and $1 \%$ penicillin-streptomycin $(\mathrm{P} / \mathrm{S})$ in a humidified atmosphere with $5 \% \mathrm{CO}_{2}$ at $37^{\circ} \mathrm{C}$. For the experiments, cells were pretreated with or without various concentrations of GJ $(5,10$, $25 \mu \mathrm{M})$ for $24 \mathrm{~h}$ and then exposed to LPS $(1 \mu \mathrm{g} / \mathrm{ml})$ for $12 \mathrm{~h}$. Additionally, p38 activation was conducted with hesperetin treatment $(2 \mu \mathrm{M}$, cat. no. HY-N0168; MedChemExpression) for $2 \mathrm{~h}$ at $37^{\circ} \mathrm{C}$ prior to other treatments.

Enzyme-linked immunosorbent assay (ELISA). After treatment, the culture medium was collected and processed for ELISA. Culture medium $(100 \mu \mathrm{l})$ was reacted with the following ELISA kits: PGE2 (cat. no. SEKH-0414; Beijing Solarbio Science \& Technology Co., Ltd.), TNF- $\alpha$ (cat. no. SEKH-0047; Beijing Solarbio Science \& Technology Co., Ltd.), IL-1 $\beta$ (cat. no. SEKH-0002; Beijing Solarbio Science \& Technology Co., Ltd.) and IL-18 (cat. no. SEKH-0028; Beijing Solarbio Science \& Technology Co., Ltd.) according to the manufacturers' protocols. The absorbance was measured at $450 \mathrm{~nm}$ using a microplate reader (Molecular Devices, LLC).

Assessment of NO. After treatment, the culture medium was collected and processed for the Griess assay. Culture medium $(50 \mu \mathrm{l})$ was mixed with an equal volume of Griess reagent (Beijing Solarbio Science \& Technology Co., Ltd.) for $10 \mathrm{~min}$ at $37^{\circ} \mathrm{C}$ in the dark. The absorbance was measured at $540 \mathrm{~nm}$ using a microplate reader (Molecular Devices, LLC).

Western blot analysis. After treatment, cells were harvested and lysed in ice-cold RIPA buffer (cat. no. P0013E; Beyotime Institute of Biotechnology) and the supernatant was collected. The protein concentration was measured using a BCA protein assay kit (cat. no. P0010; Beyotime Institute of Biotechnology). Proteins $(40 \mu \mathrm{g})$ were separated by $12 \%$ SDS-PAGE and transferred onto polyvinylidene difluoride membranes. Membranes were blocked with 5\% non-fat milk for $1 \mathrm{~h}$ at room temperature and incubated with primary antibodies at $4^{\circ} \mathrm{C}$ overnight, followed by incubation with HRP-conjugated goat anti-rabbit IgG (1:1,000; cat. no. A0208; Beyotime Institute of Biotechnology) at $37^{\circ} \mathrm{C}$ for $2 \mathrm{~h}$. Protein bands were visualized using the ECL assay kit (cat. no. P0018AM; Beyotime Institute of Biotechnology). The density of each band was normalized to the expression of the housekeeping gene GAPDH. ImageJ v1.8.0 (National Institutes of Health) was used for semi-quantification.

Immunofluorescence analysis. Cells were fixed in $2 \%$ paraformaldehyde (cat. no. P0099; Beyotime Institute of Biotechnology) for $15 \mathrm{~min}$ and permeabilized in $0.1 \%$ Triton X-100 (cat. no. ST797; Beyotime Institute of Biotechnology) for $20 \mathrm{~min}$ at room temperature, followed by incubation with $2 \%$ bovine serum albumin (BSA; cat. no. ST025; Beyotime Institute of Biotechnology) at room temperature for $60 \mathrm{~min}$ before proceeding to immunostaining. Then, cells were incubated with anti-p65 antibody (1:500; cat. no. AF1234; Beyotime Institute of Biotechnology) overnight at $4^{\circ} \mathrm{C}$ and Alexa Fluor 488-conjugated goat anti-rabbit IgG (1:250; cat. no. A0423; Beyotime Institute of Biotechnology) for 5 min at room temperature. DAPI (cat. no. C1005; Beyotime Institute of Biotechnology) was used to co-stain nuclei for $5 \mathrm{~min}$ at room temperature. Fluorescence was observed using a fluorescent microscope (Leica Microsystems, Inc.).

p38 kinase activity assay. The plates were coated with $50 \mu \mathrm{l} /$ well p38 substrate ATF-2 and stored at $4^{\circ} \mathrm{C}$ overnight. Then, plates were blocked with blocking buffer [BB, $0.05 \%$ Tween-20, $0.025 \%$ BSA and $0.02 \% \mathrm{NaN} 3$ in TBS] for a further $30 \mathrm{~min}$ at room temperature. Samples were diluted in a kinase buffer (KB), which contained $12 \mathrm{ng} / 50 \mu \mathrm{l}$ p38 MAPK, $50 \mathrm{mM}$ Tris ( $\mathrm{pH} 7.5$ ), $10 \mathrm{mM} \mathrm{MgCl} 2,10 \mathrm{mM}$ b-glycerolphosphate, $100 \mu \mathrm{g} / \mathrm{ml}$ BSA, $1 \mathrm{mM}$ dithiothreitol, $0.1 \mathrm{mM} \mathrm{Na}_{3} \mathrm{VO}_{4}$ and $100 \mu \mathrm{M}$ ATP. Each dilution was pipetted into the wells and incubated for $1 \mathrm{~h}$ at $37^{\circ} \mathrm{C}$. After the incubation, $50 \mu \mathrm{l} \mathrm{p}$-ATF-2 (Thr69/71) antibody (1:1,000, cat. no. 61584; Cell Signaling Technology, Inc.) was added into each well for $4 \mathrm{~h}$ at room temperature and then TMB substrate (200 $\mu$ l; cat. no. P0209; Beyotime Institute of Biotechnology) was added in the presence of peroxide-labeled conjugates for $10 \mathrm{~min}$ at room temperature. The reaction was measured with an ELISA reader.

Statistical analysis. Statistical analysis was performed with SPSS 11.0 (SPSS, Inc.). All data are expressed as the means \pm SD. Experiments were performed independently in triplicate. One-way analysis of variance followed by Tukey's post hoc test was used to determine significant differences between multiple groups. $\mathrm{P}<0.05$ was considered to indicate a statistically significant difference.

\section{Results}

GJ inhibits LPS-induced production of cytokines in SW982 cells. To determine the effects of GJ (Fig. 1A) on inflammatory 
A

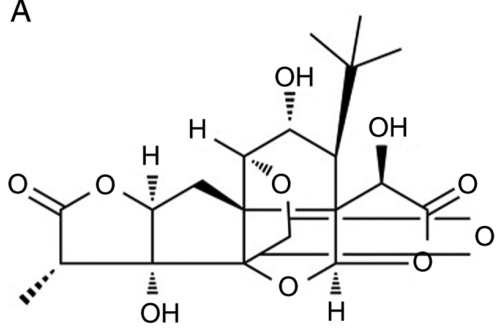

C

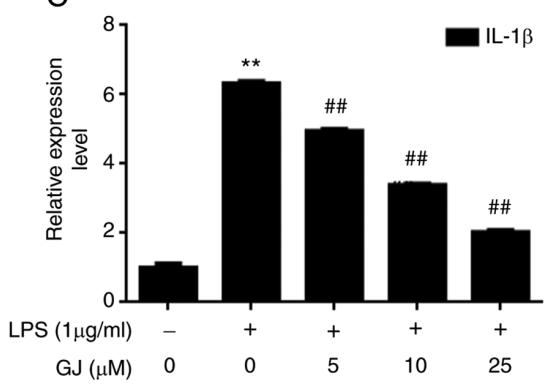

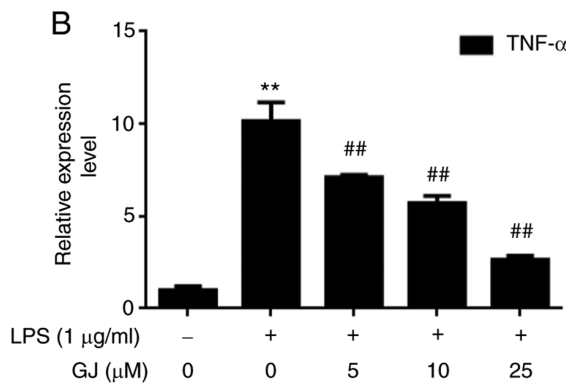

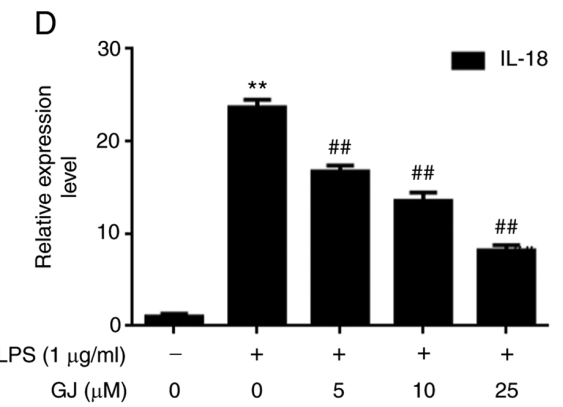

Figure 1. Relative expression levels of TNF- $\alpha$, IL-1 $\beta$ and IL-18 in SW982 cells treated with LPS and/or GJ. Cells were pre-treated with or without various concentrations of GJ $(5,10,25 \mu \mathrm{M})$ for $24 \mathrm{~h}$ and then exposed to LPS $(1 \mu \mathrm{g} / \mathrm{ml})$ for $12 \mathrm{~h}$. (A) The chemical structure of GJ. (B) The expression level of TNF- $\alpha$ was evaluated by ELISA. (C) The expression level of IL- $1 \beta$ was evaluated by ELISA. (D) The expression level of IL-18 was evaluated by ELISA. All experiments were performed in triplicate and the results were obtained from three independent studies. ${ }^{* *} \mathrm{P}<0.01$ vs. control; ${ }^{\# \#} \mathrm{P}<0.01 \mathrm{vs}$. LPS alone. TNF, tumor necrosis factor; IL, interleukin; LPS, lipopolysaccharide; GJ, ginkgolide J; ELISA, enzyme-linked immunosorbent assay.

cytokines induced by LPS stimulation, the expression levels of TNF- $\alpha$, IL-1 $\beta$ and IL-18 were assessed in SW982 cells. As revealed in Fig. 1B-D, the expression levels of TNF- $\alpha$, IL-1 $\beta$ and IL-18 were strongly induced by LPS stimulation, with an approximate 5 to 10 -fold increase. However, GJ pretreatment significantly attenuated the effect of LPS, in a dose-dependent manner. The results indicated that there is a robust effect of GJ against inflammation in LPS-treated SW982 cells.

GJ inhibits LPS-induced activation of NF- $\kappa B / N L R P 3$ signaling in SW982 cells. To determine whether the inhibitory effect of GJ on cytokine production was associated with NF- $\kappa \mathrm{B} / \mathrm{NLRP} 3$ signaling regulation, the expression levels of multiple factors that occur during this signaling were assessed in SW982 cells. As revealed in Fig. 2A and B, the upregulated expression levels of NLRP3, pro-caspase-1, caspase-1, pro-IL-1 $\beta$ and IL-1 $\beta$ as well as nucleus translocation of NF- $\kappa \mathrm{B}$ were observed in cells with LPS stimulation. However, GJ pretreatment notably attenuated the effect of LPS, in a dose-dependent manner. The results indicated that the anti-inflammatory effects of GJ were also associated with the inactivation of the NF- $\mathrm{B} / \mathrm{NLRP} 3$ signaling pathway in LPS-treated SW982 cells.

GJ inhibits LPS-induced activation of PGE2/COX-2 and iNOS/NO in SW982 cells. To determine whether GJ exerted inhibitory effects on PGE2/COX-2 and iNOS/NO signaling, the expression levels of PGE2, COX-2, iNOS and NO were assessed in SW982 cells. As revealed in Fig. 3A-C, the expression levels of PGE2, COX-2, iNOS and NO were notably upregulated in cells that underwent LPS stimulation. However, GJ pretreatment notably attenuated the effect of LPS, in a dose-dependent manner. The results indicated that the anti-inflammatory effects of GJ were associated with the suppression of PGE2/COX-2 and iNOS/NO expression in LPS-treated SW982 cells.

GJ inhibits LPS-induced upregulation and activation of p38 kinase in SW982 cells. To determine whether p38 MAPK was involved in the anti-inflammatory effects of GJ, the phosphorylation of p38 as well as p38 kinase activity were assessed in SW982 cells. As revealed in Fig. 4A and B, LPS stimulation notably induced the phosphorylation of p38 as well as enhanced the kinase activity of p38 in SW982 cells. However, GJ pretreatment markedly attenuated these effects. The results indicated that p38 kinase may be involved in the anti-inflammatory effects of GJ in LPS-treated SW982 cells.

p38 activation attenuates the effects of GJ on pro-inflammatory mediators in SW982 cells. To determine whether p38 MAPK contributed to the anti-inflammatory effects of GJ, p38 was activated in SW982 cells. As revealed in Fig. 5A and B, p38 activator (hesperetin) treatment induced the phosphorylation and activation of p38 in cells treated with LPS combined with GJ treatment, and this attenuated the anti-inflammatory effects of GJ by induction of cytokines, PGE2 and NO production (Fig. 6). The results indicated that p38 kinase contributed to the anti-inflammatory effects of GJ in LPS-treated SW982 cells.

\section{Discussion}

LPS, the main endotoxin component of gram-negative bacterial cell walls, is known for its inducive effect that elicits a strong immune response in the host (17). It stimulates cells to produce pro-inflammatory factors, such as PGE2, free radicals and cytokines, and thus leads to marked secondary inflammation in tissues $(18,19)$. Currently, LPS is used to 
A

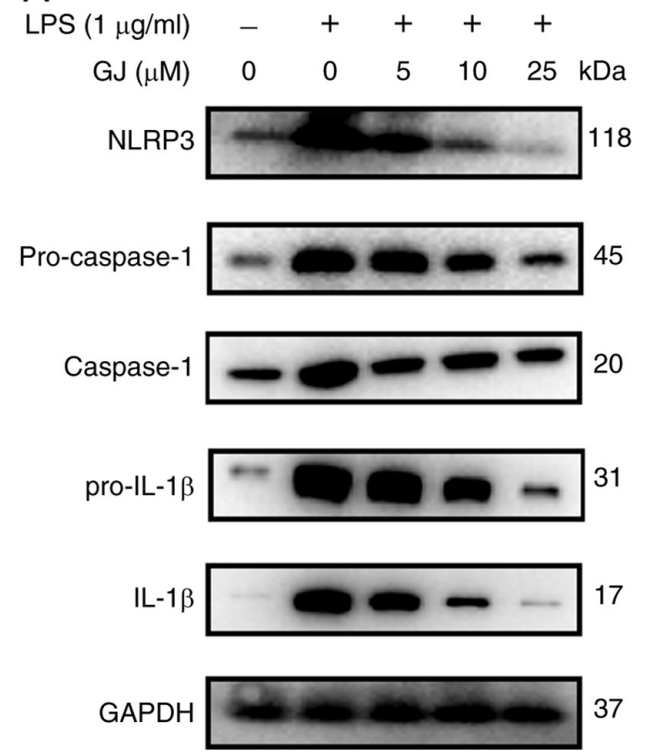

B
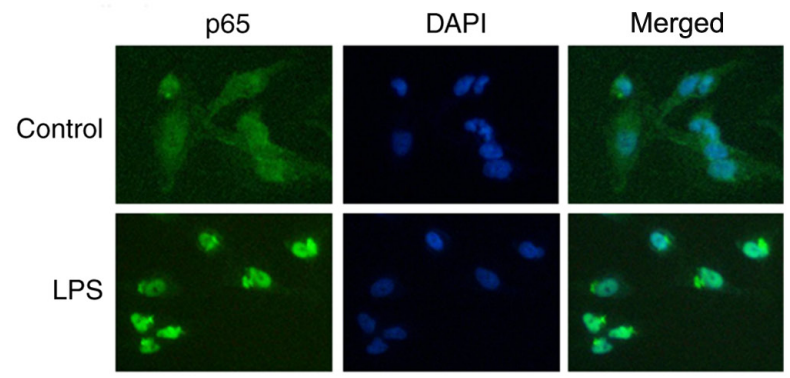

GJ $(25 \mu \mathrm{M})$
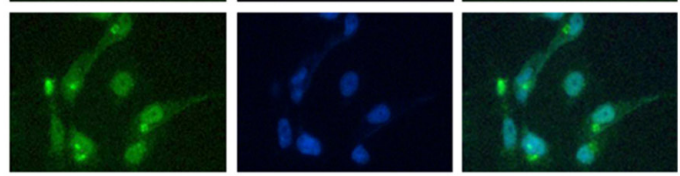

Figure 2. Activation of NF- $\mathrm{BB} / \mathrm{NLRP} 3$ signaling pathway in SW982 cells treated with LPS and/or GJ. Cells were pre-treated with or without various concentrations of GJ $(5,10,25 \mu \mathrm{M})$ for $24 \mathrm{~h}$ and then exposed to LPS $(1 \mu \mathrm{g} / \mathrm{ml})$ for $12 \mathrm{~h}$. After incubation, the activation of NF- $\mathrm{kB} / \mathrm{NLRP} 3$ signaling pathway was evaluated by western blot analysis and fluorescence assay. (A) Representative western blots of NLRP3 signaling are presented. (B) The fluorescence assay of NF- $\mathrm{KB}$ signaling was assessed. All experiments were performed in triplicate and the results were obtained from three independent studies. NF- $\mathrm{\kappa B}$, nuclear factor-kB; NLRP3, NLR family pyrin domain containing 3; LPS, lipopolysaccharide; GJ, ginkgolide J.
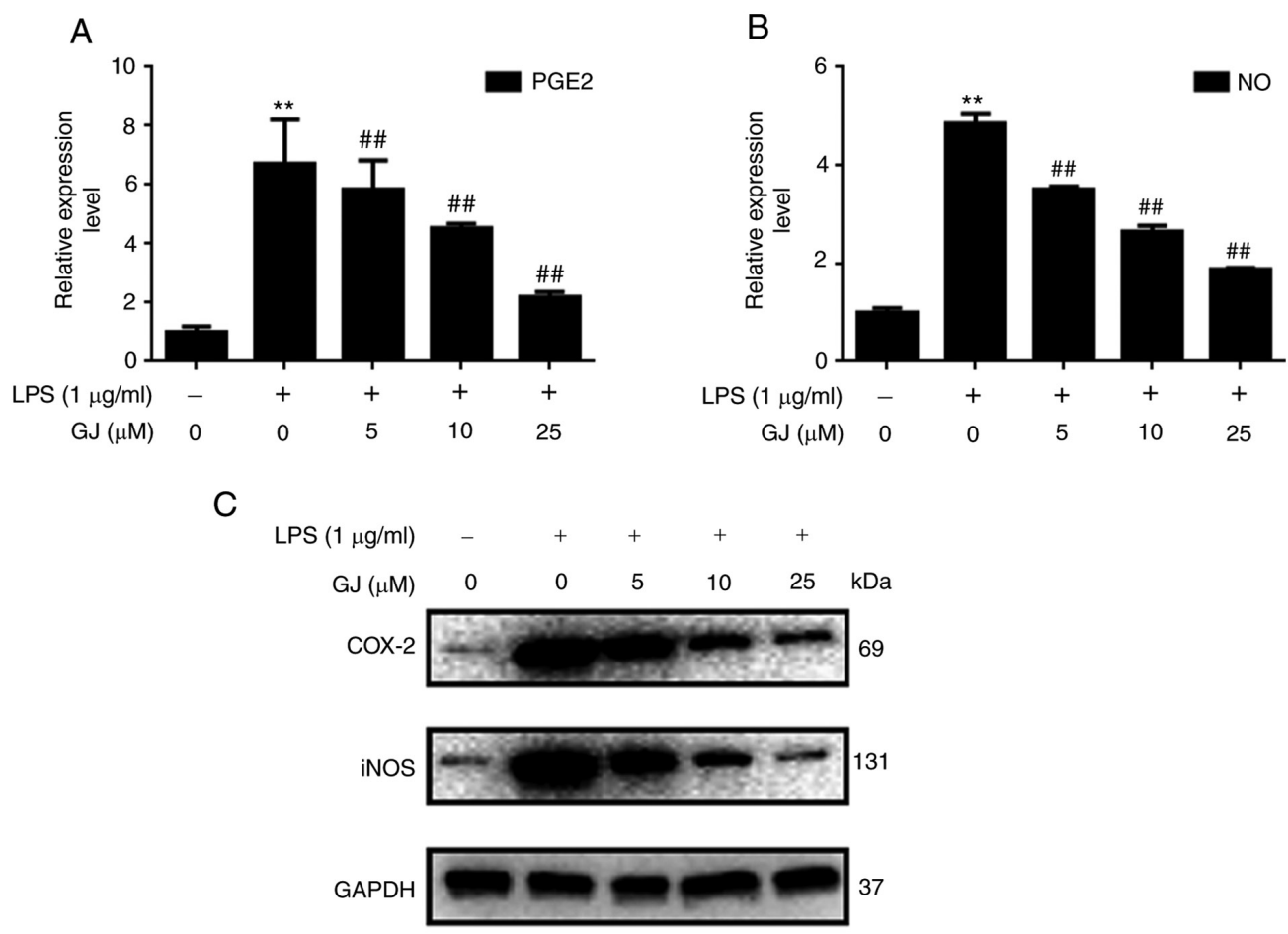

Figure 3. Relative expression levels of PGE2/COX-2 and iNOS/NO in SW982 cells treated with LPS and/or GJ. Cells were pre-treated with or without various concentrations of GJ $(5,10,25 \mu \mathrm{M})$ for $24 \mathrm{~h}$ and then exposed to LPS $(1 \mu \mathrm{g} / \mathrm{ml})$ for $12 \mathrm{~h}$. (A) The expression level of PGE2 was evaluated by ELISA. (B) The expression level of $\mathrm{NO}$ was evaluated by Griess assay. (C) The expression levels of COX-2 and iNOS were evaluated by western blot analysis. All experiments were performed in triplicate and the results were obtained from three independent studies. ${ }^{* *} \mathrm{P}<0.01$ vs. control; ${ }^{\# \#} \mathrm{P}<0.01$ vs. LPS alone. PGE2, prostaglandin E2; COX-2, cyclooxygenase-2; iNOS, inducible nitric oxide synthase; NO, nitric oxide; LPS, lipopolysaccharide; GJ, ginkgolide J.

establish transient synovitis-osteoarthritis models of RA for therapeutic research (20). Alsaleh et al (21) constructed a model using LPS-activated RA FLS to identify miRNAs that could play a role in the anti-inflammatory response. Jin et al (22) used LPS-activated RA FLS as in vitro model to identify the anti-inflammatory effect of hyperoside against LPS-induced cell proliferation and migration, cytokine production and MMP-9 secretion. Ginkgolide and bilobalide are major trilactone 
A

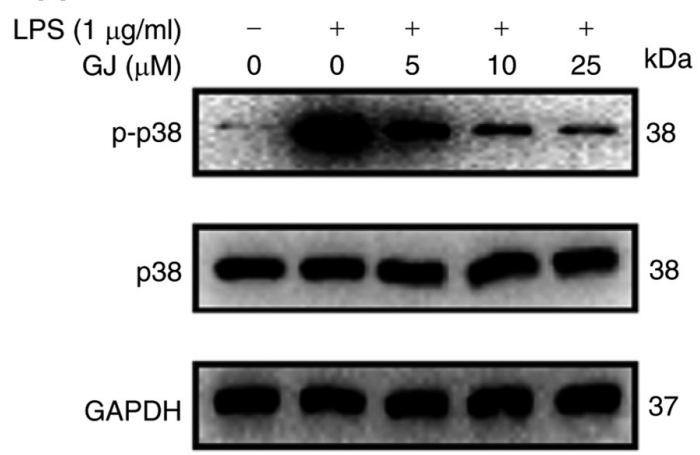

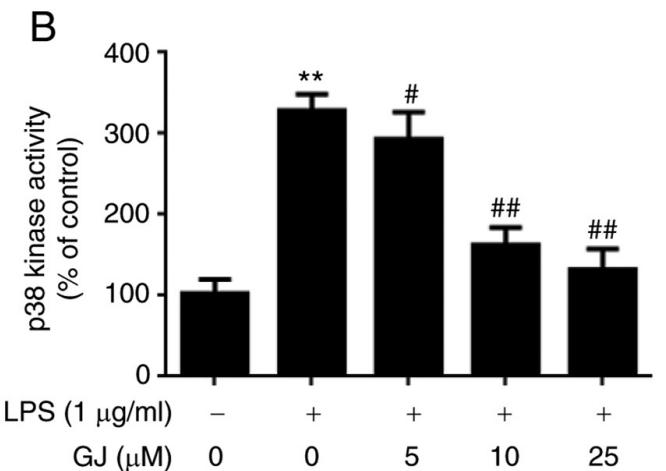

Figure 4. Activation of p38 signaling pathway in SW982 cells treated with LPS and/or GJ. Cells were pre-treated with or without various concentrations of GJ $(5,10,25 \mu \mathrm{M})$ for $24 \mathrm{~h}$ and then exposed to LPS $(1 \mu \mathrm{g} / \mathrm{ml})$ for $6 \mathrm{~h}$. After incubation, the activation of $\mathrm{p} 38$ signaling pathway was evaluated. (A) The expression levels of p-p38 and p38 were assessed by western blot analysis. (B) The p38 kinase activity was assessed by p38 kinase assay. ${ }^{* *} \mathrm{P}<0.01$ vs. control; ${ }^{~} \mathrm{P}<0.05$ and ${ }^{\# \prime} \mathrm{P}<0.01$ vs. LPS alone. All experiments were performed in triplicate and the results were obtained from three independent studies. LPS, lipopolysaccharide; GJ, ginkgolide J; p-, phosphorylated.

A

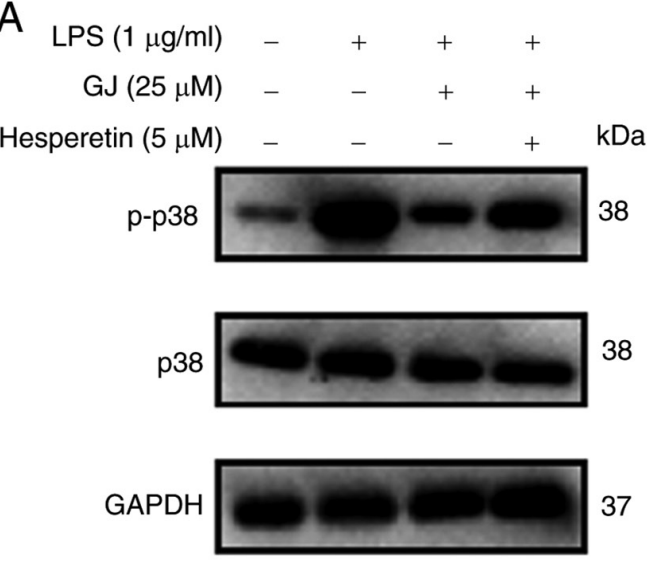

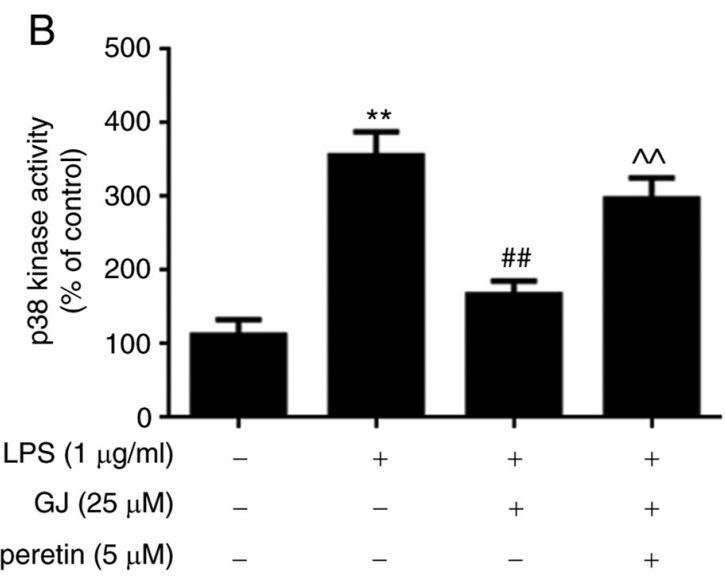

Hesperetin $(5 \mu \mathrm{M})$

Figure 5. Effect of p38 activator on p38 signaling pathway in SW982 cells treated with LPS and/or GJ. Cells were treated with indicated drugs and the activation of $\mathrm{p} 38$ signaling pathway was evaluated. (A) The expression levels of p-p38 and p38 were assessed by western blot analysis. (B) The p38 kinase activity was assessed by $\mathrm{p} 38$ kinase assay. ${ }^{* *} \mathrm{P}<0.01$ vs. control; ${ }^{\# \#} \mathrm{P}<0.01$ vs. LPS alone; ${ }^{\wedge} \mathrm{P}<0.01 \mathrm{vs}$. LPS + GJ. All experiments were performed in triplicate and the results were obtained from three independent studies. p-, phosphorylated; LPS, lipopolysaccharide; GJ, ginkgolide J.

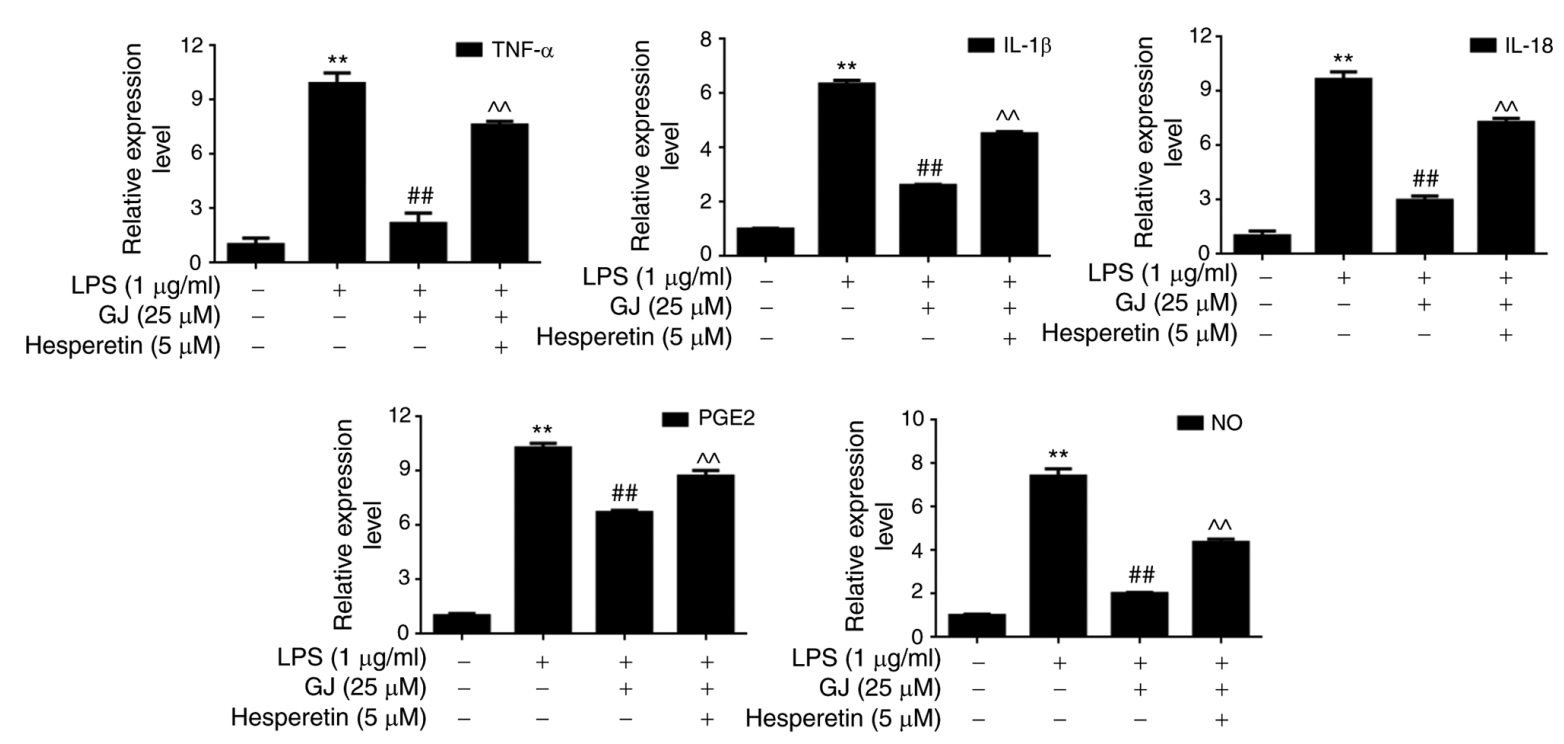

Figure 6. p38 activation on the effect of GJ on pro-inflammatory mediators in SW982 cells. Cells were treated with indicated drugs and the expression levels of PGE2 and NO as well as cytokines including TNF- $\alpha$, IL-1 $\beta$ and IL-18 were evaluated by ELISA assay. ${ }^{* *} \mathrm{P}<0.01$ vs. control; ${ }^{\# \#} \mathrm{P}<0.01$ vs. LPS alone; ${ }^{\wedge} \mathrm{P}<0.01$ vs. LPS + GJ. All experiments were performed in triplicate and the results were obtained from three independent studies. GJ, ginkgolide J; PGE2, prostaglandin E2; NO, nitric oxide; TNF, tumor necrosis factor; IL, interleukin; ELISA, enzyme-linked immunosorbent assay; LPS, lipopolysaccharide. 
constituents of Ginkgo biloba leaves and have characteristic powerful anti-inflammatory properties (10). Although several studies examining the anti-inflammatory effects of GA, GB and GC have been reported (11-15); to date, there has been little research exploring the relationship between GJ and RA inflammation, and the exact mechanism of the anti-inflammatory effect of GJ remains obscure. In the present study, using LPS-activated human synovial cells SW982, it was revealed that GJ pretreatment could attenuate LPS-induced production of pro-inflammatory mediators such as cytokines, PGE2 and NO.

NO, produced from iNOS enzymes, is upregulated in the process of inflammation and has pro-inflammatory and regulatory effects. iNOS/NO signaling was previously revealed to be most strongly activated in the synovial lining layer, subsynovium, vascular smooth muscle and chondrocytes from patients with RA (23). PGE2/COX-2 signaling also contributed to LPS-induced FLS activation, which then resulted in a release of pro-inflammatory cytokines such as TNF- $\alpha$, IL- $1 \beta$ and IL-18 (24). In addition, activation of NF- $\kappa$ B/NLRP3 signaling also occurred following LPS stimulation and contributed to a release of IL-1 $\beta$ and IL-18 (25). Activation of the three important signaling pathways was significantly attenuated by GJ pretreatment in a dose-dependent manner, indicating that $\mathrm{GJ}$ exerted its anti-inflammatory effects mainly against TNF- $\alpha / \mathrm{IL}-1 \beta / \mathrm{IL}-18 / \mathrm{NF}-\kappa \mathrm{B} / \mathrm{NLRP} 3, \mathrm{PGE} 2 / \mathrm{COX}-2$ and iNOS/NO signaling pathways.

p38, also called cytokinin-specific binding protein (CSBP), can be activated under inflammatory and stress stimuli, and participates in autophagy, apoptosis and cell differentiation (26). Accumulating evidence suggests that $\mathrm{p} 38$ plays an important role in the process of inflammation. p38 is involved in the production of proinflammatory mediators such as TNF- $\alpha$,

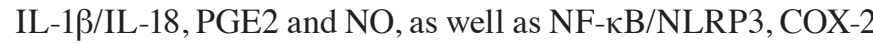
and iNOS (27). The p38 signaling pathway has been strongly implicated in the pathological process of RA, which contributes to the excessive production of pro-inflammatory mediators in FLS, and then the destruction of bone and cartilage. Thus, p38 signaling is considered as a promising target for new drug development for RA treatment (28). In the present study, the phosphorylation of p38 occurred in FLS with LPS stimulation, and it was attenuated by GJ pretreatment. In addition, p38 activator (hesperetin) treatment reversed the protective effect of GJ. The present data indicated that GJ exerted its effect by targeting p38 signaling, and then inhibited the production of pro-inflammatory mediators.

In conclusion, the results demonstrated the protective effect and detailed mechanism of GJ on LPS-treated SW982 human synovial cells, which was achieved through the suppression of the p38-dependent inflammatory signaling pathways TNF- $\alpha / \mathrm{IL}-1 \beta / \mathrm{IL}-18 / \mathrm{NF}-\kappa \mathrm{B} / \mathrm{NLRP} 3, \mathrm{PGE} 2 / \mathrm{COX}-2$ and iNOS/NO induced by LPS treatment. However, numerous questions remain to be answered. For example, in vivo experiments are required to visualize the effect of GJ against the inflammatory response. The present and a future in vivo study may contribute to the pharmaceutical potential of GJ and its derivatives in therapy for RA.

\section{Acknowledgements}

Not applicable.

\section{Funding}

The present study was supported by the Young Talent's Subsidy Project in Science and Education of the Department of Public Health of Jiangsu Province (grant no. QNRC2016627), the Six Talent Peaks Project of Jiangsu Province (grant no. WSW-047), and the Six-one Scientific Research Project (grant no. LGY2019087).

\section{Availability of data and materials}

The datasets used and/or analyzed during the current study are available from the corresponding author on reasonable request.

\section{Authors' contributions}

JW, XZ and FZ designed the experiments. YZ, YC and YL carried out the experiments. $\mathrm{YZ}, \mathrm{YC}$ and $\mathrm{KW}$ analyzed the experimental results. YZ and YC wrote the manuscript. All authors read and approved the final manuscript. JW and XZ confirm the authenticity of all the raw data.

\section{Ethics approval and consent to participate}

Not applicable.

\section{Patient consent for publication}

Not applicable.

\section{Competing interests}

The authors declare that they have no competing interests.

\section{References}

1. Pisetsky DS and Ward MM: Advances in the treatment of inflammatory arthritis. Best Pract Res Clin Rheumatol 26: 251-261, 2012.

2. Kim Y, Oh HC, Park JW, Kim IS, Kim JY, Kim KC, Chae DS, Jo WL and Song JH: Diagnosis and treatment of inflammatory joint disease. Hip Pelvis 29: 211-222, 2017.

3. Firestein GS and McInnes IB: Immunopathogenesis of rheumatoid arthritis. Immunity 46: 183-196, 2017.

4. Tak PP and Breedveld FC: Current perspectives on synovitis. Arthritis Res 1: 11-16, 1999.

5. Yoshitomi H: Regulation of immune responses and chronic inflammation by fibroblast-like synoviocytes. Front Immunol 10: 1395-1395, 2019.

6. Zuo W, Yan F, Zhang B, Li J and Mei D: Advances in the studies of Ginkgo biloba leaves extract on aging-related diseases. Aging Dis 8: 812-826, 2017.

7. Nash KM and Shah ZA: Current perspectives on the beneficial role of Ginkgo biloba in neurological and cerebrovascular Disorders. Integr Med Insights 10: 1-9, 2015.

8. Le Bars PL and Kastelan J: Efficacy and safety of a Ginkgo biloba extract. Public Health Nutr 3 (4a): 495-499, 2000.

9. Xiao G, Lyu M, Wang Y, He S, Liu X, Ni J, Li L, Fan G, Han J, Gao X, et al: Ginkgo flavonol glycosides or Ginkgolides tend to differentially protect myocardial or cerebral ischemia-reperfusion injury via regulation of TWEAK-Fn14 signaling in heart and brain. Front Pharmacol 10: 735-735, 2019.

10. Jaracz S, Malik S and Nakanishi K: Isolation of ginkgolides A, B, C, J and bilobalide from G. biloba extracts. Phytochemistry 65: 2897-2902, 2004.

11. Li Y, Wu Y, Yao X, Hao F, Yu C, Bao Y, Wu Y, Song Z, Sun Y, Zheng L, et al: Ginkgolide A Ameliorates LPS-induced inflammatory responses in vitro and in vivo. Int J Mol Sci 18: 794, 2017. 
12. Li C, Liu K, Liu S, Aerqin Q and Wu X: Role of Ginkgolides in the inflammatory immune response of neurological diseases: A review of current literatures. Front Syst Neurosci 14: 45, 2020

13. Wu F, Shi W, Zhou G, Yao H, Xu C, Xiao W, Wu J and Wu X: Ginkgolide B functions as a determinant constituent of Ginkgolides in alleviating lipopolysaccharide-induced lung injury. Biomed Pharmacother 81: 71-78, 2016.

14. Zhang H, Shi Q, Nan W, Wang Y, Wang S, Yang F and Li G: Ginkgolide B and bilobalide promote the growth and increase $\beta$-catenin expression in hair follicle dermal papilla cells of American minks. Biofactors 45: 950-958, 2019.

15. Vitolo O, Gong B, Cao Z, Ishii H, Jaracz S, Nakanishi K, Arancio O, Dzyuba SV, Lefort R and Shelanski M: Protection against beta-amyloid induced abnormal synaptic function and cell death by Ginkgolide J. Neurobiol Aging 30: 257-265, 2009.

16. Yoshino $\mathrm{S}$ and Ohsawa $\mathrm{M}$ : The role of lipopolysaccharide injected systemically in the reactivation of collagen-induced arthritis in mice. Br J Pharmacol 129: 1309-1314, 2000.

17. Bertani B and Ruiz N: Function and Biogenesis of Lipopolysaccharides. EcoSal Plus 8: 10.1128/ecosalplus. ESP-0001-2018, 2018.

18. Chang CF, Chau YP, Kung HN and Lu KS: The lipopolysaccharide-induced pro-inflammatory response in RAW264.7 cells is attenuated by an unsaturated fatty acid-bovine serum albumin complex and enhanced by a saturated fatty acid-bovine serum albumin complex. Inflamm Res 61: 151-160, 2012.

19. Yücel G, Zhao Z, El-Battrawy I, Lan H, Lang S, Li X, Buljubasic F, Zimmermann WH, Cyganek L, Utikal J, et al: Lipopolysaccharides induced inflammatory responses and electrophysiological dysfunctions in human-induced pluripotent stem cell derived cardiomyocytes. Sci Rep 7: 2935-2935, 2017.

20. Cope PJ, Ourradi K, Li Y and Sharif M: Models of osteoarthritis: The good, the bad and the promising. Osteoarthritis Cartilage 27: 230-239, 2019
21. Alsaleh G, Suffert G, Semaan N, Juncker T, Frenzel L, Gottenberg JE, Sibilia J, Pfeffer S and Wachsmann D: Bruton's tyrosine kinase is involved in miR-346-related regulation of IL-18 release by lipopolysaccharide-activated rheumatoid fibroblast-like synoviocytes. J Immunol 182: 5088-5097, 2009.

22. Jin XN, Yan EZ, Wang HM, Sui HJ, Liu Z, Gao W and Jin Y: Hyperoside exerts anti-inflammatory and anti-arthritic effects in LPS-stimulated human fibroblast-like synoviocytes in vitro and in mice with collagen-induced arthritis. Acta Pharmacol Sin 37: 674-686, 2016.

23. Grabowski PS, Wright PK, Van 't Hof RJ, Helfrich MH, Ohshima H and Ralston SH: Immunolocalization of inducible nitric oxide synthase in synovium and cartilage in rheumatoid arthritis and osteoarthritis. Br J Rheumatol 36: 651-655, 1997.

24. Kawashima M, Ogura N, Akutsu M, Ito K and Kondoh T: The anti-inflammatory effect of cyclooxygenase inhibitors in fibroblast-like synoviocytes from the human temporomandibular joint results from the suppression of PGE2 production. J Oral Pathol Med 42: 499-506, 2013.

25. Fu Q, Gao Y, Zhao H, Wang Z and Wang J: Galangin protects human rheumatoid arthritis fibroblast like synoviocytes via suppression of the NF $\kappa B / N L R P 3$ pathway. Mol Med Rep 18: 3619-3624, 2018

26. Herlaar E and Brown Z: p38 MAPK signalling cascades in inflammatory disease. Mol Med Today 5: 439-447, 1999.

27. Schieven GL: The p38alpha kinase plays a central role in inflammation. Curr Top Med Chem 9: 1038-1048, 2009.

28. Yong HY, Koh MS and Moon A: The p38 MAPK inhibitors for the treatment of inflammatory diseases and cancer. Expert Opin Investig Drugs 18: 1893-1905, 2009.

(i) (9) This work is licensed under a Creative Common

c) AY NG NO Attribution-NonCommercial-NoDerivatives 4.0 International (CC BY-NC-ND 4.0) License. 